\title{
RADEMACHER'S INFINITE PARTIAL FRACTION CONJECTURE IS (almost certainly) FALSE
}

\author{
ANDREW V. SILLS AND DORON ZEILBERGER
}

\begin{abstract}
"It is a capital mistake to theorise before one has data. Insensibly one begins to twist facts to suit theories, instead of theories to suit facts."-Sherlock Holmes to Dr. Watson 22 p. 63].

AbSTRaCT. In his book Topics in Analytic Number Theory, Hans Rademacher conjectured that the limits of certain sequences of coefficients that arise in the ordinary partial fraction decomposition of the generating function for partitions of integers into at most $N$ parts exist and equal particular values that he specified. Despite being open for nearly four decades, little progress has been made toward proving or disproving the conjecture, perhaps in part due to the difficulty in actually computing the coefficients in question. In this paper, we provide a fast algorithm for calculating the Rademacher coefficients, a large amount of data, direct formulas for certain collections of Rademacher coefficients, and overwhelming evidence against the truth of the conjecture. While the limits of the sequences of Rademacher coefficients do not exist (the sequences oscillate and attain arbitrarily large positive and negative values), the sequences do get very close to Rademacher's conjectured limits for certain (predictable) indices in the sequences.
\end{abstract}

\section{IMPORTANT Note}

This article is accompanied by the Maple package HANS, downloadable from http://www.math.rutgers.edu/ ${ }^{2}$ zeilberg/tokhniot/HANS .

The "front" of this article, http://www.math.rutgers. edu/ ${ }^{\sim}$ zeilberg/mamarim/mamarimhtml/hans.html contains lots of supporting input and output files.

\section{INTRODUCTION}

Let $p_{N}(n)$ denote the number of partitions of the integer $n$ into at most $N$ parts. The generating function of $p_{N}(n)$,

$$
F_{N}(x):=\sum_{n \geq 0} p_{N}(n) x^{n}=\prod_{j=1}^{N} \frac{1}{1-x^{j}},
$$

Date: October 22, 2018.

2010 Mathematics Subject Classification. Primary 11 Y99.

A. V. S. thanks the Center for Discrete Mathematics and Theoretical Computer Science (DIMACS) for its hospitality during his July 2011 stay, which led to this collaboration with D. Z..

D. Z. is supported in part by a grant of the National Science Foundation. 
may be decomposed into partial fractions:

$$
\prod_{j=1}^{N} \frac{1}{1-x^{j}}=\sum_{k=1}^{N} \sum_{\substack{0 \leq h<k \\ \operatorname{gcd}(h, k)=1}} \sum_{l=1}^{\lfloor N / k\rfloor} \frac{C_{h, k, l}(N)}{\left(x-e^{2 \pi i h / k}\right)^{l}} .
$$

We shall refer to the $C_{h, k, l}(N)$ defined by (1.1) as the Rademacher coefficients.

Near the end of his posthumously published masterpiece Topics in Analytic Number Theory [5, p. 302], Hans Rademacher made the following conjecture:

Rademacher's Conjecture. For all integers $h, k, l$ such that $0 \leq h<k, \operatorname{gcd}(h, k)=$ 1 and $l \geq 1, \lim _{N \rightarrow \infty} C_{h, k, l}(N)$ exists and equals

$$
R_{h, k, l}:=-2 \pi\left(\frac{\pi}{12}\right)^{3 / 2} \frac{e^{\pi i(s(h, k)+2 h l / k)}}{k^{5 / 2}} \Delta_{\alpha}^{l-1} L_{3 / 2}\left(-\frac{\pi^{2}}{6 k^{2}}(\alpha+1)\right),
$$

evaluated at $\alpha=\frac{1}{24}$, where $s(h, k)=\sum_{\mu=1}^{k-1}\left(\frac{\mu}{k}-\left\lfloor\frac{\mu}{k}\right\rfloor-\frac{1}{2}\right)\left(\frac{h \mu}{k}-\left\lfloor\frac{h \mu}{k}\right\rfloor-\frac{1}{2}\right)$ is the Dedekind sum, $\Delta_{\alpha}$ is the forward difference operator, so that

$$
\Delta_{\alpha}^{j} f(\alpha)=\sum_{h=0}^{j}(-1)^{h}\left(\begin{array}{l}
j \\
h
\end{array}\right) f(\alpha+j-h)
$$

and

$$
L_{3 / 2}\left(-y^{2}\right)=-\frac{1}{2 \sqrt{\pi} y^{2}}\left(2 \cos (2 y)-\frac{\sin (2 y)}{y}\right) .
$$

If the Rademacher conjecture would have been true then it would have followed that

$$
\begin{gathered}
\lim _{N \rightarrow \infty} C_{0,1,1}(N)=R_{0,1,1}\left(=-\frac{6}{25}\left(1+\frac{2 \sqrt{3}}{5 \pi}\right)=-0.292927573960 \ldots\right), \\
\lim _{N \rightarrow \infty} C_{0,1,2}(N)=R_{0,1,2}\left(=\frac{144}{1225}+\frac{5616}{42875 \pi}=0.1897670688440 \ldots\right), \\
\lim _{N \rightarrow \infty} C_{1,2,1}(N)=R_{1,2,1}\left(=-\frac{2 \sqrt{6}}{25}\left(\cos \frac{5 \pi}{12}-\frac{12}{5 \pi} \sin \frac{5 \pi}{12}\right)=0.093882853484 \ldots\right) .
\end{gathered}
$$

Remark 1.1. The floating-point approximation for the value of $R_{0,1,1}$ stated by Rademacher [5. p. 302] was erroneous, as were the exact values of $R_{0,1,2}$ and $R_{1,2,1}$ (for the latter he gave exactly one half of the correct value). These erroneous values were quoted, without correction, by Andrews [1, p. 388].

Rademacher supplied (with one error) a table of values for $C_{0,1,1}(N), C_{0,1,2}(N)$, and $C_{1,2,1}(N)$ for $N=1,2,3,4,5$, and in fact these values are not too far off from his conjectured " $N=\infty$ " cases.

Rademacher began work on the book in which this conjecture appeared [5] no later than 1944 and was still working on it at the inception of his final illness. Thus as the final version was edited and published by Rademacher's students Emil Grosswald, Joseph Lehner, and Morris Newman, after Rademacher's death in 1969, we will never know whether Rademacher came to doubt the truth of the conjecture after he had written it down. However, George Andrews reports that Rademacher discussed the conjecture in a course he taught at the University of Pennsylvania during the 1961-1962 academic year. 
In 4, Augustine Munagi considered a different type of partial fraction decomposition called $q$-partial fractions, and proved a special case of the analog of the Rademacher conjecture, relative to the $q$-partial fraction decomposition.

We should also note that the first to cast doubts on the Rademacher conjecture were Jane Friedman and Leon Ehrenpreis. Ehrenpreis [3, p. 317] stated, "If one attempts to carry out the usual type of partial fraction decomposition of the partition function term-by-term, it is difficult to compute the coefficients. My student, Jane Friedman, spent a great deal of time trying to apply computer algorithm methods to compare the coefficients with those of Rademacher... Unfortunately, the computer study proved inconclusive."

In this article, we present overwhelming evidence against this conjecture, taken literally, but we will present ample evidence for a modified conjecture. Additionally, we will present a fast algorithm for generating the Rademacher coefficients, and formulas for a selection of particular Rademacher coefficients.

\section{Empirical EVIDENCE AGAinst the RADEMACHeR CONJECTURE}

2.1. The actual behavior of the sequences $C_{0,1, l}(N), l=1,2,3, \ldots$ At http://www.math.rutgers.edu/ zeilberg/mamarim/mamarimhtml/hans.html , there are links to various files including

- the sequences $C_{0,1, l}(N)$ for $1 \leq l \leq 10$ and $1 \leq N \leq 850$, in Maple-readable format,

- the sequences of floating point approximations to $C_{0,1, l}(N)$ for $1 \leq l \leq 40$ and $1 \leq N \leq 1000$, in Maple-readable format.

Figures 1 and 2 are graphical summaries of $C_{0,1,1}(N)$.

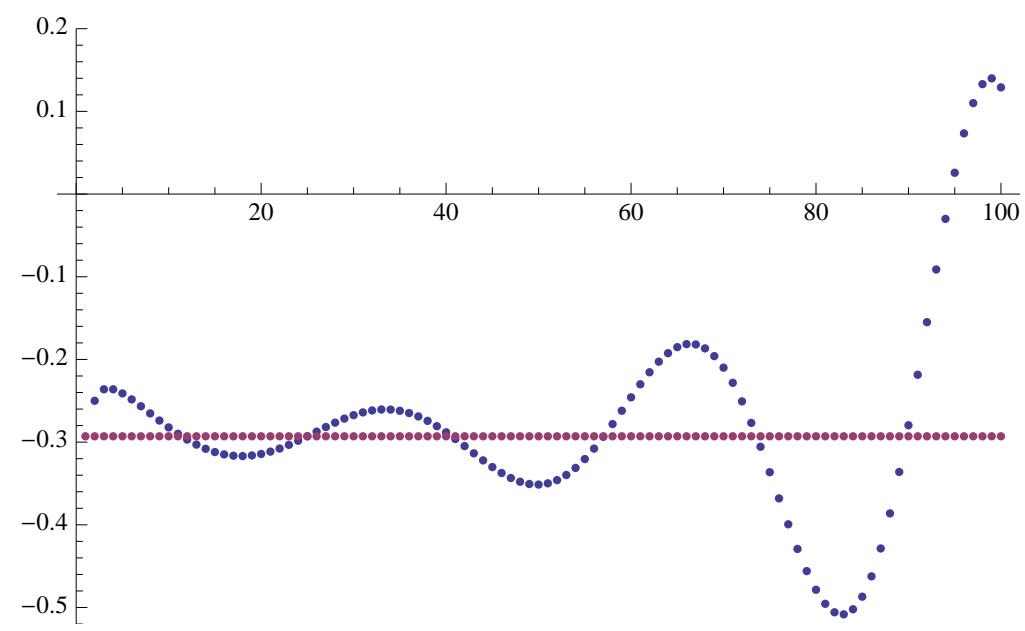

Figure 1. Graph of $C_{0,1,1}(N)$ for $N$ from 1 to 100, together with the line $y=R_{0,1,1}$

Note that $C_{0,1,1}(25)$ differs from $R_{0,1,1}$, Rademacher's conjectured value of $C_{0,1,1}(\infty)$, by less than 0.000032 , but then things go down hill (for the conjecture) from there, and get particularly bad after about $n=150$. Numerical evidence points to the 


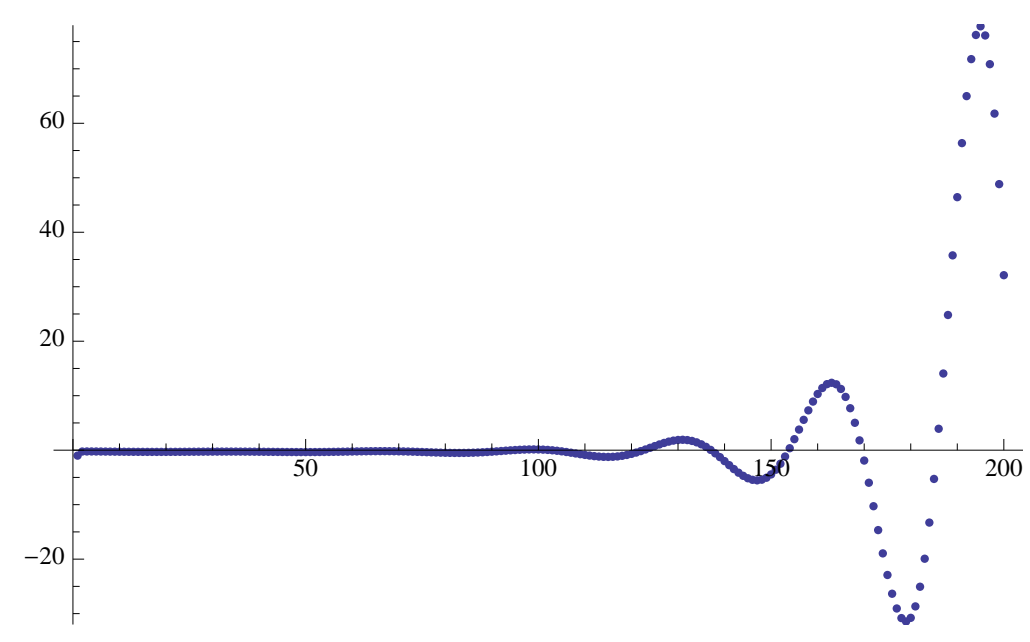

Figure 2. Graph of $C_{0,1,1}(N)$ for $N$ from 1 to 200 .

sequence $C_{0,1,1}(N)$ oscillating and attaining arbitrarily large positive and negative values. The same thing is true for other sequences $C_{h, k, l}(N)$; see the output file at http://www . math.rutgers.edu/ zeilberg/tokhniot/oHANS11 .

By examining the graphs in Figures 1 and 2 and the associated numerical data, it seems reasonable to state the following alternative conjecture:

Conjecture 2.1. $C_{0,1,1}(N)$ is an oscillating function of $N$ of "period" 32 , with local maxima (resp. local minima) that attain arbitrarily large positive (resp. negative) values as $N$ increases.

By "period 32" we mean that the peaks and valleys, eventually, recur at a period of 32. We also noticed, numerically, that the elevations and depths of successive peaks and valleys roughly grows exponentially with a factor around 8 . Specifically, $C_{0,1,1}(N)$ has local maxima at $N=3,4,33,66,99,131,163,195,227,259,291,323$, $355,387,419,451,483,515,547,579,611,643,675,707,739,771, \ldots$ The ratio of consecutive local maxima is

$$
\begin{gathered}
\{1,1.103504574,0.6965131681,-0.7709983810,13.63072659,6.485614677, \\
6.289519948,6.547018652,6.785098547,6.992410281,7.161220864,7.301859590, \\
7.420337150,7.521483398,7.608822684,7.684977203,7.751953124, \\
7.811301903,7.864245038,7.911756412,7.954622120,7.993483579, \\
8.028869316,8.061218737,8.090900135, \ldots\}
\end{gathered}
$$

$C_{0,1,1}(N)$ has a local minimum half way between each local maximum, at $N=$ $18,50,83,115,147,179,211,243,275,307,339,371,403,435,467,499,531,563,595$, $627,659,691,723,755,787, \ldots$

More precise conjectured asymptotics for $C_{0,1, l}(N)$ for $l$ between 1 and 15 can be gotten from

http://www.math.rutgers.edu/ ${ }^{\sim}$ zeilberg/tokhniot/oHANS10 . 
It appears that all $C_{0,1, l}(N)$ have a "period" of 32 . The locations of the local maxima and minima of $C_{0,1, l}(N)$, from $N=99$ until $N=803$ occur when $N \equiv 3$ $(\bmod 32)$ and $N \equiv 19(\bmod 32)$ respectively. For $N>803$, they become $N \equiv 2$ (mod 32$)$ and $N \equiv 18(\bmod 32)$ respectively. This gives (very meager) evidence of a "shifting of the perihelion", but one would need to go much further to investigate this. More generally, for $C_{0,1, l}(N)$, and for $100 \leq N \leq 800$ these locations are congruent to $12-9 l(\bmod 32)$, and $28-9 l(\bmod 32)$ respectively. Probably these too would eventually get shifted (ever so slowly).

2.2. How to compute the sequences $C_{0,1, l}(N)$ fast. If you use the definition of $C_{0,1,1}(N)$, or Andrews's formula [1, p. 388, Theorem 1], you can't go very far, even with Maple. Rademacher calculated $C_{0,1,1}(N)$ for $N=1,2,3,4,5$, presumably by hand, and made an error in the $N=5$ case. Andrews [1, p. 388], who had access to a computer algebra system in 2003, corrected Rademacher's error at $N=5$ and extended the list to $N=6,7,8$.

We need to be more clever. A fast recurrence for $C_{0,1, l}(N)$ can be derived as follows. Since

$$
\prod_{j=1}^{N} \frac{1}{1-x^{j}}=\sum_{l=1}^{N} \frac{C_{0,1, l}(N)}{(x-1)^{l}}+\ldots
$$

we can multiply both sides by $(x-1)^{N}$ and get

$$
(x-1)^{N} \prod_{j=1}^{N} \frac{1}{1-x^{j}}=\sum_{r=0}^{N-1} D_{r}(N)(x-1)^{r}+\ldots .
$$

Once we know $D_{r}(N)$, we can find $C_{0,1, l}(N)$, since they equal $D_{N-l}(N)$. It remains to find a fast recurrence for $D_{r}(N)$.

By definition, we have:

$$
\frac{1-x^{N}}{x-1}\left(\sum_{r=0}^{\infty} D_{r}(N)(x-1)^{r}\right)=\sum_{r=0}^{\infty} D_{r}(N-1)(x-1)^{r} .
$$

Letting $z=x-1$, this is:

$$
\frac{1-(z+1)^{N}}{z}\left(\sum_{r=0}^{\infty} D_{r}(N) z^{r}\right)=\sum_{r=0}^{\infty} D_{r}(N-1) z^{r} .
$$

By the binomial theorem,

$$
-\left(\sum_{a=0}^{N-1}\left(\begin{array}{c}
N \\
a+1
\end{array}\right) z^{a}\right)\left(\sum_{r=0}^{\infty} D_{r}(N) z^{r}\right)=\sum_{r=0}^{\infty} D_{r}(N-1) z^{r} .
$$

Equating coefficients of $z^{r}$ we get:

$$
N D_{r}(N)+\sum_{a=1}^{r}\left(\begin{array}{c}
N \\
a+1
\end{array}\right) D_{r-a}(N)=-D_{r}(N-1) .
$$

And finally:

$$
D_{r}(N)=-\frac{D_{r}(N-1)}{N}-\sum_{a=1}^{r} \frac{1}{N}\left(\begin{array}{c}
N \\
a+1
\end{array}\right) D_{r-a}(N)
$$

This is implemented in procedure $\mathrm{C} 01(1, \mathrm{~N})$ of HANS. 
The same argument leads to efficient recurrences for $C_{h, k, l}(N)$, except that now we have to distinguish between the case when $N$ is divisible by $k$ and when it is not, yielding two different recurrences. This is implemented in procedure $\operatorname{Chk} l \mathrm{~N}(\mathrm{~h}, \mathrm{k}, \mathrm{l}, \mathrm{N})$ of HANS.

2.3. $C_{1,2,1}(N)$. The values of $C_{1,2,1}(N)$ for $1 \leq N \leq 700$, in both exact rational form and approximate floating point form are provided at

http://www . math.rutgers . edu/ ${ }^{\sim}$ zeilberg/tokhniot/oHANS3 .

Graphical summaries are provided in Figures 3 and 4 . As the graphs make clear, it is better to regard $C_{1,2,1}(N)$ as two separate subsequences, $C_{1,2,1}(2 n)$ and $C_{1,2,1}(2 n+1)$. For each subsequence, we see behavior that is similar to that of $C_{0,1, l}(N)$.

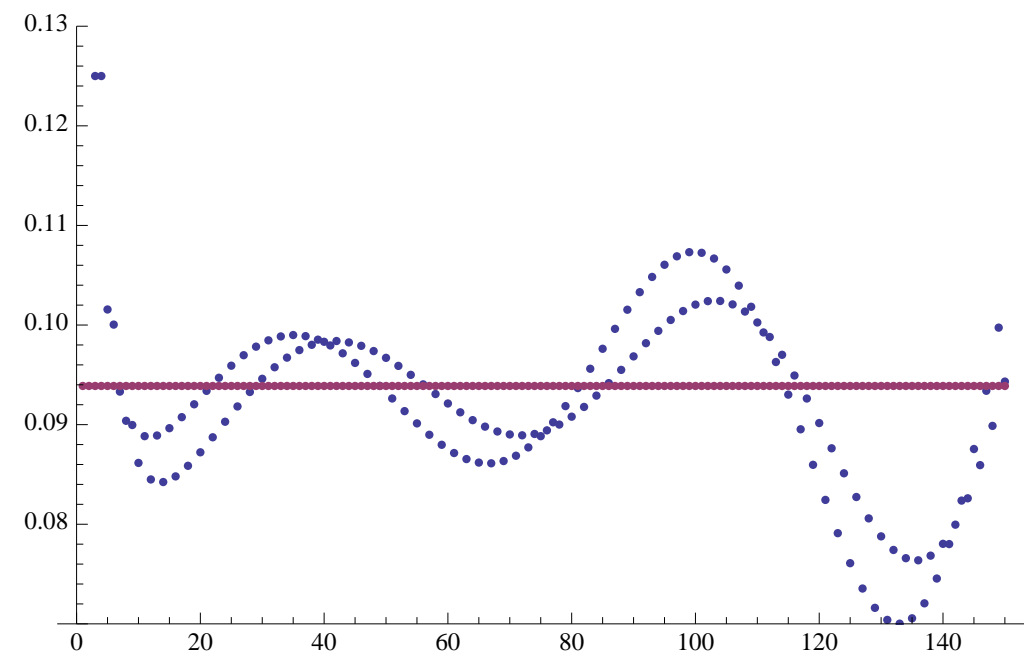

Figure 3. Graph of $C_{1,2,1}(N)$ for $N$ from 1 to 150 , together with the line $y=R_{1,2,1}$

\section{3. "Top DOWn" FORmulas For the Rademacher Coefficients}

3.1. $C_{0,1, l}(N)$. As Rademacher already pointed out, it seems hopeless to get a closed-form formula for $C_{h, k, l}(N)$ for $l=1,2, \ldots$, but if you work your way down from the "top", one can conjecture, and then rigorously prove explicit formulas for $C_{h, k, N-r}(N)$, that alas, get increasingly more complicated as $r$ gets larger.

\section{Conjecture 3.1.}

$$
C_{0,1, N-r}(N)=\frac{(-1)^{N+r}}{4^{r} N ! r !} P_{0,1, N-r}(N),
$$

where, for $r>0, P_{0,1, N-r}(N)$ is a convex, alternating, monic polynomial of degree $2 r$ whose only real roots are 0 and 1 .

Theorem 3.2. Explicit formulas for the $P_{0,1, N-r}(N)$ of Conjecture 3.1 may be given for any specific $r$, in particular, we have

$$
P_{0,1, N}(N)=1
$$




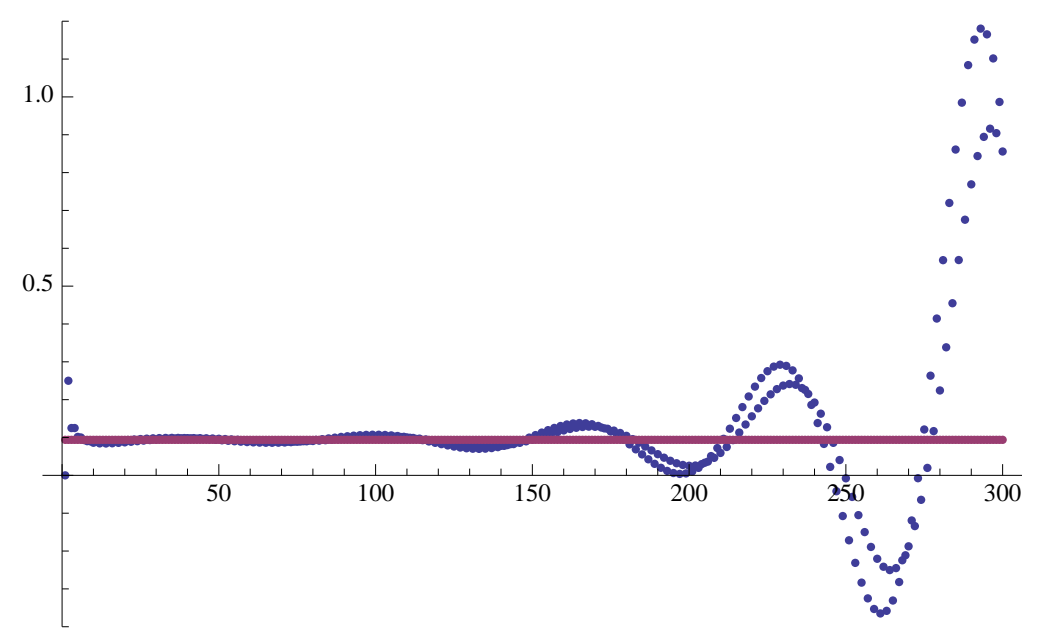

Figure 4. Graph of $C_{1,2,1}(N)$ for $N$ from 1 to 300, together with the line $y=R_{1,2,1}$

$$
\begin{gathered}
P_{0,1, N-1}(N)=N^{2}-N \\
P_{0,1, N-2}(N)=N^{4}-\frac{22 N^{3}}{9}+\frac{13 N^{2}}{3}-\frac{26 N}{9} \\
P_{0,1, N-3}(N)=N^{6}-\frac{13 N^{5}}{3}+\frac{43 N^{4}}{3}-25 N^{3}+\frac{98 N^{2}}{3}-\frac{56 N}{3} \\
P_{0,1, N-4}(N)=N^{8}-\frac{20 N^{7}}{3}+\frac{862 N^{6}}{27}-\frac{21104 N^{5}}{225}+\frac{29039 N^{4}}{135}-\frac{14548 N^{3}}{45} \\
+\frac{9892 N^{2}}{27}-\frac{42896 N}{225}
\end{gathered}
$$

Remark 3.3. Readers desiring formulas for $C_{0,1, N-r}(N)$ for $r>4$ are directed to the ChkFormula procedure in the HANS Maple package.

Remark 3.4. From the above (and additional data not reproduced here but available at the website), we may deduce that

$$
\begin{gathered}
P_{0,1, N-r}(N)=N^{2 r}-\frac{2 r^{2}+7 r}{9} N^{2 r-1}+\frac{4 r^{4}+12 r^{3}+287 r^{2}-303 r}{2 \cdot 9^{2}} N^{2 r-2} \\
-\frac{200 r^{6}-300 r^{5}+40706 r^{4}+42939 r^{3}-257509 r^{2}+173964 r}{150 \cdot 9^{3}} N^{2 r-3} \\
+ \text { lower degree terms. }
\end{gathered}
$$

For $s>1$, the coefficients of $N^{2 r-s}$ again appear to be polynomials in $r$ of degree $2 s$, whose real roots include 0 and 1 , although they are not convex and may have additional real roots. 
Proof of Theorem 3.2. Define $G_{N}:=(x-1)^{N} F_{N}(x)$. Then $G_{N}$ has a Taylor series expansion about $x=1$, whose first $N$ coefficients are the Rademacher coefficients:

$$
G_{N}=\sum_{j=0}^{N-1} C_{0,1, N-j}(N)(x-1)^{j}+\text { higher degree terms . }
$$

Clearly,

$$
\left(1-x^{N}\right) G_{N}=(x-1) G_{N-1} .
$$

Expanding $\left(1-x^{N}\right)$ on the left hand side of (3.6) as a Taylor polynomial about $x=1$, we have

$$
\begin{aligned}
\left(-\sum_{j=1}^{N}\left(\begin{array}{c}
N \\
j
\end{array}\right)(x-1)^{j}\right) & \left(\sum_{j=0}^{N-1} C_{0,1, N-j}(N)(x-1)^{j}+\text { higher degree terms }\right) \\
& =\sum_{j=1}^{N-1} C_{0,1, N-j}(N-1)(x-1)^{j}+\text { higher degree terms }
\end{aligned}
$$

Comparing the coefficients of $(x-1)^{1}$ on both sides of (3.7), we find

$$
-N C_{0,1, N}(N)=C_{0,1, N-1}(N-1) .
$$

Solving the recurrence with the initial condition $C_{0,1,1}(1)=-1$, yields

$$
C_{0,1, N}(N)=\frac{(-1)^{N}}{N !}
$$

which is 3.1.

Comparing the coefficients of $(x-1)^{2}$ on both sides of (3.7), we find, taking into account (3.8),

$$
-N C_{0,1, N-1}(N)-\left(\begin{array}{c}
N \\
2
\end{array}\right) \frac{(-1)^{N}}{N !}=C_{0,1, N-2}(N-1)
$$

with initial condition $C_{0,1,1}(2)=-\frac{1}{4}$ yields

$$
C_{0,1, N-1}(N)=\frac{(-1)^{N+1}}{4(N-2) !}
$$

which is 3.2 .

Comparing the coefficients of $(x-1)^{3}$ on both sides of (3.7), we find, taking into account (3.8) and (3.10),

$$
-N C_{0,1, N-2}(N)-\left(\begin{array}{c}
N \\
2
\end{array}\right) \frac{(-1)^{N+1}}{4(N-2) !}-\left(\begin{array}{c}
N \\
3
\end{array}\right) \frac{(-1)^{N}}{N !}=C_{0,1, N-3}(N-1)
$$

with initial condition $C_{0,1,1}(3)=-\frac{17}{72}$ yields

$$
C_{0,1, N-2}(N)=\frac{(-1)^{N}\left(9 N^{2}-13 N+26\right)}{288(N-2) !},
$$

which is 3.3 . Results for larger $r$ follow analogously. 
3.2. $C_{1,2, l}(N)$. Let us now define $\bar{G}_{N}$ analogously to $G_{N}$. Let

$$
\bar{G}_{N}:=(x+1)^{\lfloor N / 2\rfloor} F_{N}(x) .
$$

Then $\bar{G}_{N}$ has a Taylor series expansion about $x=-1$, whose first $\left\lfloor\frac{N}{2}\right\rfloor$ coefficients are the Rademacher coefficients:

$$
\bar{G}_{N}=\sum_{r=0}^{\lfloor N / 2\rfloor-1} C_{1,2,\lfloor N / 2\rfloor-r}(N)(x+1)^{r}+\text { higher degree terms . }
$$

We now abandon the use of the floor function. Notice that

$$
\left(1-x^{2 n-1}\right)\left(1-x^{2 n}\right) \bar{G}_{2 n}=(x+1) \bar{G}_{2 n-2} .
$$

Thus, by expanding the two left most factors on the left side as a Taylor series about $x=-1$,

$$
\begin{aligned}
& \left\{\sum_{r=1}^{4 n-1}(-1)^{r+1}\left[\left(\begin{array}{c}
4 n-1 \\
r
\end{array}\right)+\left(\begin{array}{c}
2 n \\
r
\end{array}\right)-\left(\begin{array}{c}
2 n-1 \\
r
\end{array}\right)\right](x+1)^{r}\right\} \\
& \times\left(\sum_{r=0}^{n-1} C_{1,2, n-r}(2 n)(x+1)^{r}+\text { higher degree terms }\right) \\
& =\sum_{r=1}^{n-1} C_{1,2, n-r}(2 n-2)(x+1)^{r}+\text { higher degree terms }
\end{aligned}
$$

By comparing coefficients of $(x+1)^{r}$ in both sides of (3.14) and solving the recurrences, we obtain formulas for $C_{1,2, n-r}(2 n)$ analogous to those for $C_{0,1, N-r}(N)$.

$$
\begin{gathered}
C_{1,2, n}(2 n)=\frac{1}{2^{2 n} n !} . \\
C_{1,2, n-1}(2 n)=\frac{n}{2^{2 n}(n-1) !} . \\
C_{1,2, n-2}(2 n)=\frac{18 n^{3}-8 n^{2}+15 n+2}{9 \cdot 2^{2 n+2}(n-1) !} .
\end{gathered}
$$

Of course, the observation $\left(1-x^{2 n}\right)\left(1-x^{2 n+1}\right) \bar{G}_{2 n+1}=(x+1) \bar{G}_{2 n-1}$ leads to analogous formulas for the $C_{1,2, n-r}(2 n+1)$, e.g.,

$$
\begin{gathered}
C_{1,2, n}(2 n+1)=\frac{1}{2^{2 n+1} n !}, \\
C_{1,2, n-1}(2 n+1)=\frac{2 n^{2}+2 n+1}{2^{2 n+2} n !}, \\
C_{1,2, n-2}(2 n+1)=\frac{18 n^{5}+46 n^{4}+61 n^{3}+53 n^{2}+29 n+9}{9 \cdot 2^{2 n+3}(n+1) !},
\end{gathered}
$$

Clearly, the same idea can be used to find formulas for $C_{h, k, n-j}(k n+r)$ for any $h, k, j, r$. This has been implemented in the procedure ChkFormula in the HANS Maple package. For those desiring automatically generated papers, containing both formulas of this type and their proofs, please use the HansTopDownAutoPaper procedure in the HANS Maple package. 


\section{Close Encounters of the Rademacher Kind}

While it appears that $\lim _{N \rightarrow \infty} C_{h, k, l}(N)$ does not exist for any $(h, k, l)$, we can nonetheless define $B_{h, k, l}$ to be the $N$ which comes closest to $R_{h, k, l}$. This is implemented in the CloseEncounters procedure in HANS.

\begin{tabular}{|c|c|r|r|}
\hline$l$ & $B_{0,1, l}$ & $\left|C_{0,1, l}\left(B_{0,1, l}\right)-R_{0,1, l}\right|$ & $\left|C_{0,1, l}\left(B_{0,1, l}\right) / R_{0,1, l}\right|$ \\
\hline 1 & 25 & 0.0003177 & 0.99989 \\
2 & 47 & 0.0001434 & 0.99924 \\
3 & 71 & 0.0000828 & 0.99991 \\
4 & 149 & 0.0000009 & 1.00001 \\
\hline
\end{tabular}

Notice that the first few values of $B_{0,1, l}$ are close to $24 l$. This motivates us to consider comparing $C_{0,1, l}(24 l)$ to $R_{0,1, l}$.

\begin{tabular}{|c|l|l|}
\hline$l$ & $\left|C_{0,1, l}(24 l)-R_{0,1, l}\right|$ & $\left|C_{0,1, l}(24 l) / R_{0,1, l}\right|$ \\
\hline 1 & 0.0053741095 & 1.018346206 \\
2 & 0.0015044594 & 1.007927400 \\
3 & 0.00033240887 & 0.996241370 \\
4 & 0.00004427030 & 1.001376635 \\
5 & 0.000011288321 & 0.9988220859 \\
6 & 0.000001686611 & 1.0006971253 \\
7 & 0.0000001275687 & 0.9997575030 \\
8 & 0.0000000110523 & 1.0000986383 \\
9 & 0.00000000239242 & 0.9999562770 \\
10 & 0.000000005333208 & 1.0000141594 \\
11 & 0.0000000187490584 & 0.9999947242 \\
12 & 0.0000000393434274 & 1.0000017401 \\
\hline
\end{tabular}

Thus we have some evidence that even though the $N$ for which $C_{0,1, l}(N)$ is closest to $R_{0,1, l}$ is not $N=24 l, C_{0,1, l}(24 l)$ seems to provide a good approximation to $R_{0,1, l}$, and the approximation seems to be improving as $l$ increases.

\section{REFERENCES}

1. G. E. Andrews, Partitions: at the interface of $q$-series and modular forms, Ramanujan J. 7 (2003) 385-400.

2. A. Conan Doyle, The Adventures of Sherlock Holmes: Adventure I-A Scandal in Bohemia, The Strand Magazine 2, no. 7 (1891) 61-75.

3. L. Ehrenpreis, Function theory for Rogers-Ramanujan-like partition identities, in A Tribute to Emil Grosswald: Number Theory and Related Analysis, ed. M. I. Knopp and M. Sheingorn, Contemporary Math 143 (1993) 259-320.

4. A. O. Munagi, The Rademacher conjecture and $q$-partial fractions, Ramanujan J. 15 (2008) 339-347.

5. H. Rademacher, Topics in Analytic Number Theory, Die Grundlehren der mathematischen Wissenschaften in Einzeldarstellungen Band 169, Springer, 1973.

Andrew V. Sills, Department of Mathematical Sciences, Georgia Southern UniverSity, 65 Georgia Avenue, Room 3008, Statesboro, Georgia 30458-8093, USA

E-mail address: ASills@GeorgiaSouthern.edu

Doron Zeilberger, Department of Mathematics, Rutgers University, Hill Center, 110 Frelinghuysen Road, Piscataway, NJ 08854-8019, USA

E-mail address: zeilberg@math.rutgers.edu 